\title{
A STUDY OF MICROCANTILEVER QUALITY FACTOR
}

\author{
Kevin Y. Yasumuraa) , Timothy D. Stowe, Eugene M. Chow, Timothy Pfafman, \\ Thomas W. Kenny \\ Departments of Applied Physics, Electrical Engineering, and Mechanical Engineering, \\ Stanford University, Stanford California 94305-4021 \\ Daniel Rugar \\ IBM Research Division, Almaden Research Center, San Jose, California 95120-6099
}

\begin{abstract}
Micromechanical cantilevers are commonly used for detection of small forces in MEMS sensors (accelerometers) and in scientific instruments (atomic force microscopes). A fundamental limit to the measurement of small forces is imposed by the mechanical analog of Johnson noise, thermomechanical noise, which is governed by dissipation of mechanical energy in the force measuring cantilever. In this paper we report on measurements of the mechanical quality factor, $Q$, for arrays of silicon nitride, polysilicon, and single crystal silicon cantilevers. By studying the dependence of the mechanical energy dissipation on the cantilever material, geometry, and surface treatments, we hope to identify the dissipation mechanisms, and offer guidelines for the design of cantilevers for measurements of small forces. Preliminary results show that $Q$ decreases with cantilever thickness indicating surface loss mechanisms. Significant improvement of the $Q$ is obtained after annealing in either $\mathrm{N}_{2}$ or forming gas.
\end{abstract}

\section{INTRODUCTION}

The majority of MEMS sensors measure forces applied to micromechanical flexures. Examples include pressure sensors which measure force on a diaphragm, and accelerometers and gyroscopes which measure inertial force on a proof mass. Because of the cost advantages associated with miniaturization, many sensors are required to measure surprisingly small forces. For example, the Analog Devices ADXL05 accelerometer features a proof mass with a mass of approximately $10^{-10} \mathrm{~kg}$ and is capable of detecting an acceleration as small as $5 \times 10^{-4} \mathrm{~g}$ in a $1 \mathrm{~Hz}$ bandwidth. This acceleration represents a force of $0.5 \mathrm{pN}$ applied to the mass.

Ordinarily, sensor performance is improved by reducing the noise of the preamplifier used to convert the physical signals to electrical signals, and by controlling other error sources such as uncompensated thermal drift. There eventually comes a point, however, where thermodynamics imposes a barrier to further sensor improvement. For the case of microcantilevers optimized for use in force detection, thermomechanical noise sets a limit to the ultimate force resolution [1].

Thermomechanical noise is a consequence of the cantilever being in thermal equilibrium with its environment (i.e., a heat bath with many microscopic degrees of freedom). Energy dissipation in the cantilever causes the stored mechanical energy to leak away and be converted to heat. The stronger the coupling between the cantilever and the heat bath, the faster the decay of cantilever motion towards thermal equilibrium and the lower the mechanical quality factor, $Q$, of the oscillating mode. Conversely, the coupling to the heat bath has the consequence that the cantilever will be subjected to constant random excitation by its interaction with the many microscopic degrees of freedom in the heat bath. This relationship between the energy dissipation and random excitation is embodied in the "fluctuation-dissipation theorem" of statistical mechanics, which applies to mechanical systems just as it applies to the Johnson noise across an electrical resistor. The net result is that the lower the mechanical $Q$ of the system, the larger the force noise.

The equipartition theorem gives a measure of how much thermal energy is in each mode of a microcantilever. The mean square vibration amplitude associated with a mode of oscillation is given by

$$
\frac{1}{2} k_{B} T=\frac{1}{2} k\left\langle x^{2}\right\rangle
$$

We can calculate the equivalent force noise associated with mechanical dissipation by imposing the requirement that random thermal excitations must produce the mean square vibration amplitude given by Eq. 1. The mean square vibration amplitude is the integral over all frequencies of the force noise spectral density multiplied by the mechanical transfer function

$$
\left\langle x^{2}\right\rangle=\int_{0}^{\infty}|G(f)| S_{F} d f,
$$

where the transfer function is

$$
G(f)=\frac{f_{0}^{2} / k}{\left(f_{\bullet}^{2}-f^{2}\right)+i\left(f f_{0} / Q\right)} .
$$

This results in a force noise spectral density of $S_{F}=4 k k_{B} T / \omega_{1} Q$. This spectral density results in a noise force in a bandwidth $B$ of

$$
F_{\text {inin }}=\sqrt{\frac{4 k k_{B} T B}{\omega_{0} Q}} .
$$

This minimum detectable force can also be expressed in terms of the cantilever dimensions: $w$ is the cantilever width, $l$ is the cantilever length, and $t$ is the cantilever thickness.

$$
F_{\min }=\left(\frac{w c^{2}}{l Q}\right)^{1 / 2}\left(k_{B} T B\right)^{1 / 2}(E \rho)^{1 / 4} \text {, }
$$

where $E$ is the modulus of elasticity and $\rho$ is the mass density of the cantilever material. From this equation for the minimum detectable force a strategy can be found to design ultrasensitive cantilevers: make them narrow, thin, and long. This strategy is effective only if high mechanical $Q$ is maintained. Unfortunately, relatively little is understood about the mechanisms responsible for intrinsic energy dissipation in micron and submicron thick microstructures.

\footnotetext{
a) Danarment mail: Department of Applied Physics, Stanford University, Stanford, CA, 94305-4021; Electronic mail: yasumura@leland.stanford.edu
} 
For a beam oscillating in a flexural mode, the stored energy is proportional to the square of the peak strain integrated over the volume of the beam. If the primary source of dissipation is due to bulk internal losses (i.e., internal friction due to lattice dislocations, impurities, two level systems, etc.) and if the dissipation is proportional to the square of the strain, as is usually the case, then the energy lost per cycle will also be proportional to the square of the peak strain integrated over the volume of the beam. Since the stored energy and the dissipation involve the same volume integrals of strain squared, their ratio, and hence $Q$, will be independent of cantilever geometry.

On the other hand, if the dissipation is not due to bulk internal losses, but rather due to surface losses [2], then the dissipation will be proportional to the square of the peak strain integrated over the surface area of the beam. After evaluation of the appropriate integrals, one finds that the $Q$ for a cantilever oscillating in its lowest order flexural mode will be proportional to thickness, $t$. This dependence on thickness would be expected for micromechanical systems with large surface-to-volume ratios.

Based on this reasoning, we have set out to study the relationship between dissipation and controllable cantilever properties such as material, geometry, and surface treatment. We have fabricated arrays of silicon nitride, polysilicon, and single crystal silicon cantilevers and performed measurements of the $Q$ of these cantilevers. In this paper we present the preliminary results from this ongoing study.

\section{CANTILEVER FABRICATION}

Three materials have been used to fabricate our cantilevers-silicon nitride, polysilicon, and single crystal silicon. The first, silicon nitride, was chosen because of its durability, ease of fabrication, and general use as a processing material. The second material, polysilicon, was chosen because of its wide use in the MEMS community as a sensor material. A number of fabrication processes rely on a top polysilicon layer from which a sensor or device is fabricated, making a study of dissipation in polysilicon resonators of wide interest. The last material, single crystal silicon, was chosen because of its expected low internal friction as exhibited by larger bulk

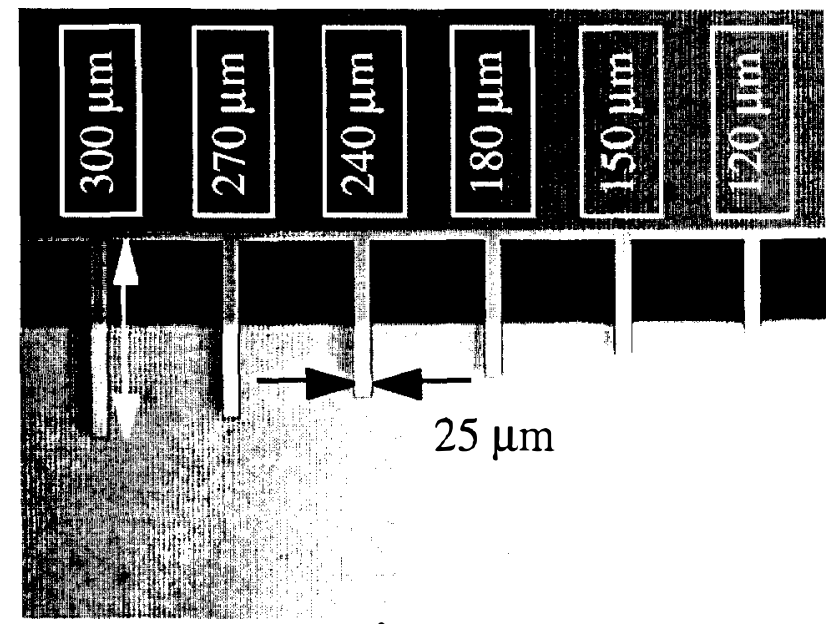

Figure 1. Array of $2000 \AA$-thick silicon nitride cantilevers. Shown is part of an array of $w=25 \mu \mathrm{m}$ cantilevers of length varying from 120 to $300 \mu \mathrm{m}$. Cantilever arrays made from silicon nitride and polysilicon use this array pattern. oscillators $[3,4]$ and low internal stress allowing for the fabrication of ultrathin cantilevers with little or no curling [5].

Silicon nitride cantilevers, as shown in Fig. 1, were fabricated from LPCVD low stress silicon nitride grown on $\langle 100\rangle$ silicon wafers. After film deposition, the cantilevers were patterned and then defined using an $\mathrm{SF}_{6}$ dry etch. A TMAH etch was then used to etch away the exposed silicon and undercut the cantilevers. Finally, critical point drying was used to release the thin cantilever structures [6].

Polysilicon cantilevers were fabricated from polysiliconon-insulator wafers. Starting with a $\langle 100\rangle$ silicon wafer, a 4000 $\AA$-thick layer of thermal oxide was grown. Next a polysilicon layer of the desired cantilever thickness was deposited. The cantilevers were then patterned using a mask pattern similar those in Fig. 1 and protective layers of low temperature oxide and silicon nitride were deposited to protect the cantilevers during backside patterning and a TMAH etch. Lastly, the topside silicon nitride layer was removed and a BOE etch was used to free the oxide encased cantilever structures. Finally, a critical point drying step was performed.

Single crystal silicon cantilevers start with $\langle 100\rangle$ SOI wafers. A thermal oxidation was performed to thin down the top silicon layer to the desired cantilever thickness. A BOE etch was then used to remove the top oxide layer exposing the top silicon layer for cantilever patterning. As in the polysilicon process, low temperature oxidation and silicon nitride deposition was then used for frontside protection and as a backside masking layer during the backside TMAH etch. The cantilevers were then released with a BOE etch followed by critical point drying to prevent cantilever stiction and curling. Fig. 2 shows a photograph of a $600 \AA$-thick single crystal silicon cantilever. Further details of the fabrication process can be found in the work of Stowe, et al. [5].

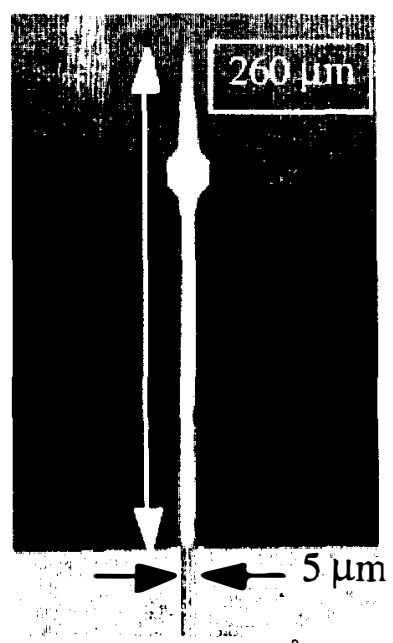

Figure 2. Photograph of a $600 \AA$-thick single crystal silicon cantilever. Arrays of such cantilevers consist of cantilevers of varying length. Shown is a cantilever with a neck width of $5 \mu \mathrm{m}$ and length of $260 \mu \mathrm{m}$. 


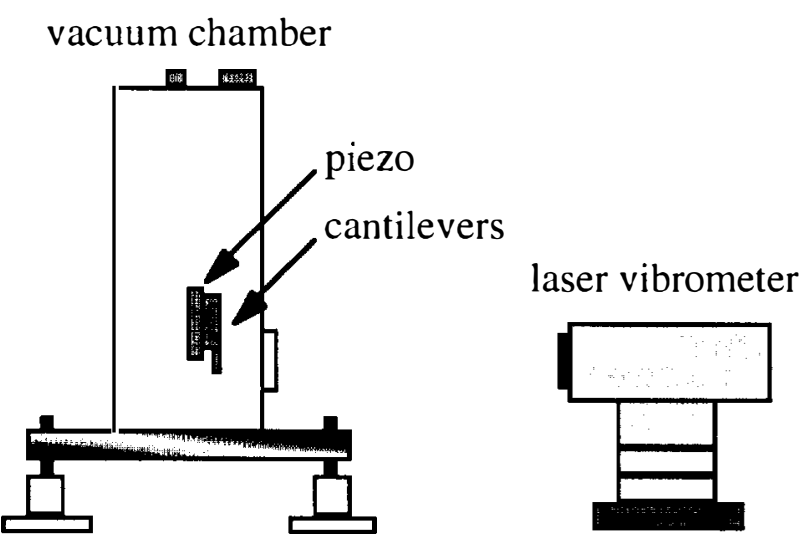

Figure 3. Diagram of the $Q$ measurement system. Shown are the vacuum chamber, piezoelectric disk stack, cantilever die, and laser doppler vibrometer.

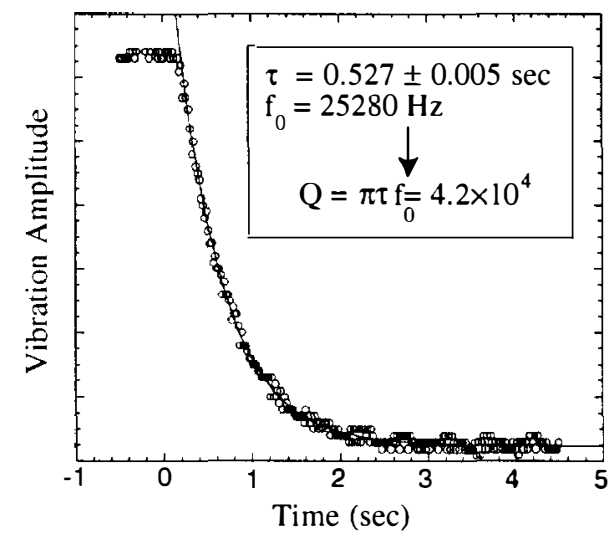

Figure 4. Sample cantilever ring-down and exponential curve fit. Cantilever is polysilicon $t=2.3 \mu \mathrm{m}, w=25 \mu \mathrm{m}$, $l=210 \mu \mathrm{m}$.

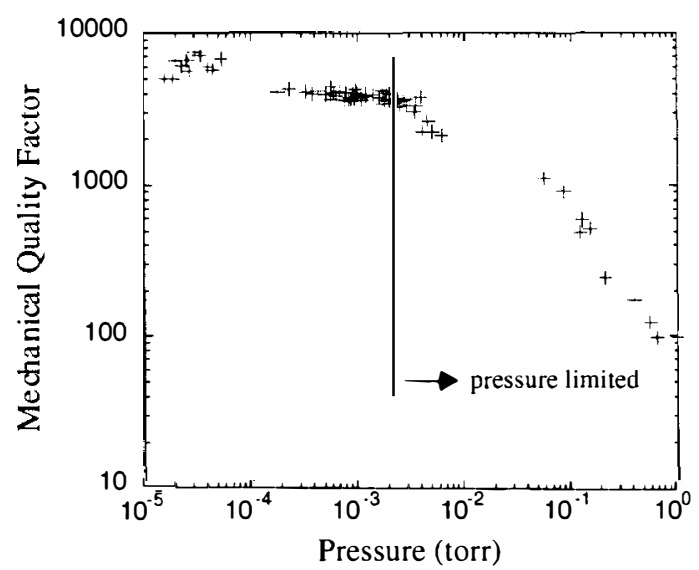

Figure 5. $Q$ vs. pressure for a $5100 \AA$-thick silicon nitride cantilever.

\section{Q MEASUREMENTS}

A diagram of the experimental system is shown in Fig. 3 . The cantilevers were placed in a vacuum chamber on a stack of piezoelectric disks. A viewport on the side of the dewar allows the use of an external laser doppler vibrometer (LDV) to measure the cantilever motion. The LDV is a commercially available system which measures the velocity dependent doppler shift of the reflected laser radiation [7].

A "free ring-down" technique was used to measure cantilever $Q$. The cantilevers were first driven on-resonance to a steady state amplitude. The drive excitation was produced by applying an oscillating voltage to the piezoelectric disks on which the cantilever die has been mounted. The drive was stopped abruptly and the cantilever motion was measured as the amplitude decayed. Figure 4 shows a typical ring-down for a polysilicon cantilever. The ring-down was then curve fit to an exponential function. From the fit, the time constant of the ring-down, $\tau$, was obtained. The decay time constant, with the cantilever resonance frequency for the first mode, allows calculation of the cantilever $Q=\pi \tau f_{0}$. The exponential decay curve fit allows us to be sure of the accuracy of each $Q$ measurement. Data presented in this paper are averages of multiple ring-down measurements for each cantilever. As shown in Fig. 4, the error for each individual cantilever ringdown measurement is on the order of a few percent.

One important source of energy dissipation in micromechanical oscillators is air damping. Figure 5 shows a measurement of the mechanical $Q$ as a function of air pressure for silicon nitride cantilevers $5100 \AA$ thick. At atmospheric pressure, the $Q$ of this cantilever was significantly suppressed by air damping. We can see that at pressures of 1 mtorr and below, the dissipation associated with air damping becomes negligible compared to intrinsic loss mechanisms. The work described in this paper was performed under vacuum at a pressure of $10^{-6}$ torr. The measurements were performed at room temperature.

For the cantilever used in Fig. 4, the repeatability and long term stability of the cantilever $Q$ was measured over a period of two hours. The results are plotted in Fig. 6. Over this two hour time span the $Q$ had a standard deviation of $1 \%$ demonstrating excellent measurement reproducibility. On the other hand, retesting of cantilevers after they have been exposed to laboratory air for several days can show much larger $Q$ variability.

Measurements of $Q$ for arrays of silicon nitride cantilevers were carried out for thicknesses of $2000 \AA$, $5100 \AA$, and $7000 \AA$, lengths from 120 to $300 \mu \mathrm{m}$, and widths of 10 and $25 \mu \mathrm{m}$. Fig. 7 shows data for three arrays of silicon nitride cantilevers of thicknesses $2000 \AA, 5100 \AA$, and $7000 \AA$. All of the cantilevers in Fig. 7 had a width of $10 \mu \mathrm{m}$. Each of the points in Fig. 7 are the result of the averaging of multiple ring-down measurements for each cantilever. Several trends are clear from this data. First, the $Q$ is roughly independent of the cantilever length. Both bulk (volume) and surface dependent dissipative processes are expected to produce length independent $Q$ 's. However, there is an increase in the mechanical $Q$ as the thickness of the cantilever increases. This behavior is most clearly seen in 


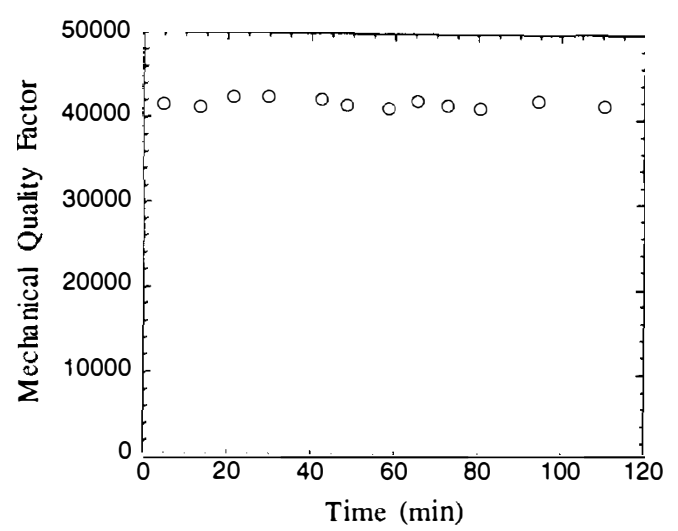

Figure 6. Mechanical $Q$ vs. Time for a polysilicon cantilever $(t=2.3 \mu \mathrm{m}, w=25 \mu \mathrm{m}, l=210 \mu \mathrm{m})$ showing $a$ $1 \%$ standard deviation in the mechanical $Q$ over a two hour time span.

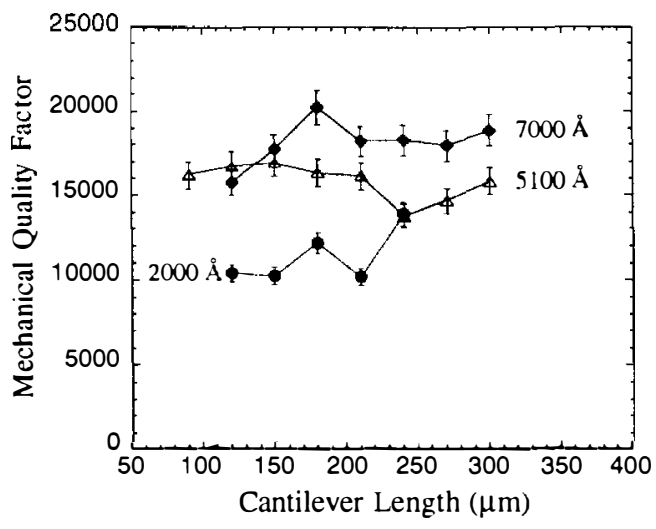

Figure 7. Mechanical quality factor vs. cantilever length for silicon nitride cantilevers of thicknesses $2000 \AA, 5100 \AA$, and $7000 \AA$. Cantilever width is $10 \mu \mathrm{m}$ for all three thicknesses.

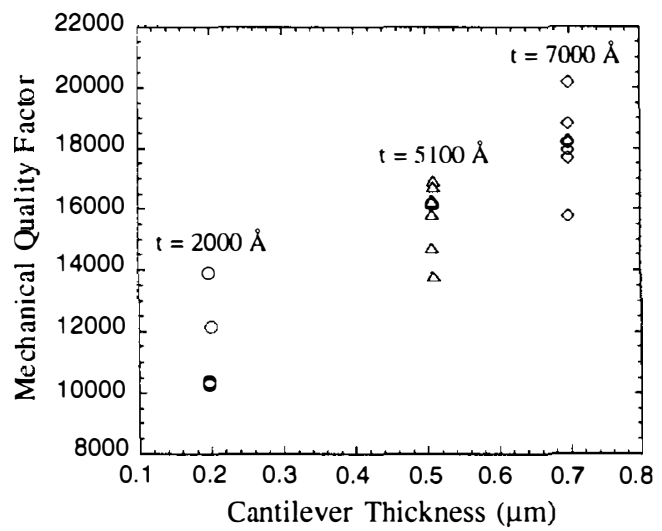

Figure 8. Mechanical quality factor vs. cantilever thickness for three silicon nitride cantilever thicknesses $(2000 \AA, 5100 \AA, 7000 \AA)$.
Fig. 8, where we have replotted the data as a function of cantilever thickness. The strong thickness dependence is indicative of surface loss mechanisms, as discussed earlier.

Figure 9 shows data for silicon nitride cantilevers of thickness $5100 \AA$ and two widths of 10 and $25 \mu \mathrm{m}$. The $Q$ 's of these cantilevers are independent of the cantilever width. Just as the cantilever $Q$ is expected to be length independent for both volume and surface dissipative processes, the cantilever $Q$ should also be independent of cantilever width.

Single crystal silicon cantilevers show geometrical dependence similar to the nitride devices. Figure 10 shows data for single crystal silicon cantilevers of thicknesses $600 \AA$ and $1700 \AA$. Consistent with the data in Figs. 7 and 8, we see that the $Q$ is roughly independent of cantilever length and proportional to thickness.

Figure 11 shows a compilation of data from our work and from the literature for single crystal silicon oscillators of varying thickness at $T=4 \mathrm{~K}$. As in the case of silicon nitride cantilevers, the $Q$ of single crystal silicon oscillators is highly dependent on the oscillator thickness, suggesting surface dominated loss mechanisms. Because this plot has data for different kinds of oscillators it should not be over interpreted, but the general trend is evident-an increase in the mechanical $Q$ as the oscillator dimensions increase. Included in Fig. 11 is data for ultrathin silicon cantilevers [5,9], doubly supported high frequency silicon resonators [8], commercially available silicon cantilevers [10], double torsional oscillators [11], and suspended bulk silicon crystals [12]. Shown in Fig. 11 is a line fit to the four thinnest oscillators $(t=600 \AA, 1700 \AA, 3300$ $\AA$, and $1.5 \mu \mathrm{m})$. These micron- and submicron-scale cantilevers have a roughly linear $Q$ vs. $t$ dependence. The larger oscillators deviate from this linear dependence possibly because loss mechanisms other than surface mechanisms begin to dominate and limit the $Q$ as the oscillator size increases.

In order to make quantitative comparisons of different cantilever geometries and materials, a parameter which we shall call the 'loss parameter' $\gamma$ must be introduced. Recall from Eq. 5 that the strategy to make sensitive force sensing microcantilevers was to make them narrow, thin, and long (small $k\left(\omega_{0}\right)$ while maintaining high mechanical $Q$. This general strategy tells us that $Q$ is not the best parameter to use when comparing different oscillators. Even though larger bulk silicon oscillators can obtain $Q$ 's of 600,000 at room temperature [4], the large mass and high spring constant of these oscillators make them poor force detectors. In order to determine whether a cantilever, and more generally any oscillator, would be a sensitive force measurement device, all of the cantilever dependent terms in Eq. 4 must be taken into account. By using $\gamma \equiv k / \omega_{0} Q$, we can compare cantilevers of different geometries and materials. Expressed in terms of the cantilever geometries and material properties, $\gamma$ can be expressed as

$$
\gamma=0.246 \frac{w t^{2}}{l Q}(E \rho)^{1 / 2}
$$

for the first flextural mode of a simple beam cantilever. For more complicated structures, the correct spring constant and resonance frequencies for each mode of oscillation must be used. This analysis can therefore be extended to include torsional and double torsional oscillators as well as more complex systems where an effective spring constant and resonance frequency can be either calculated or measured directly. Figure 12 shows a 


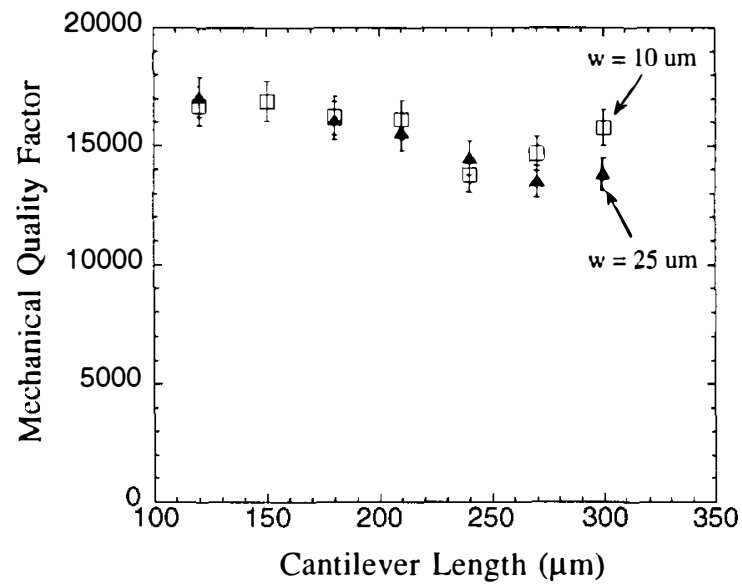

Figure 9. Mechanical quality factor vs. cantilever length for silicon nitride cantilevers of thickness $5100 \AA$. Two cantilever widths are shown, $10 \mu \mathrm{m}$ and $25 \mu \mathrm{m}$.

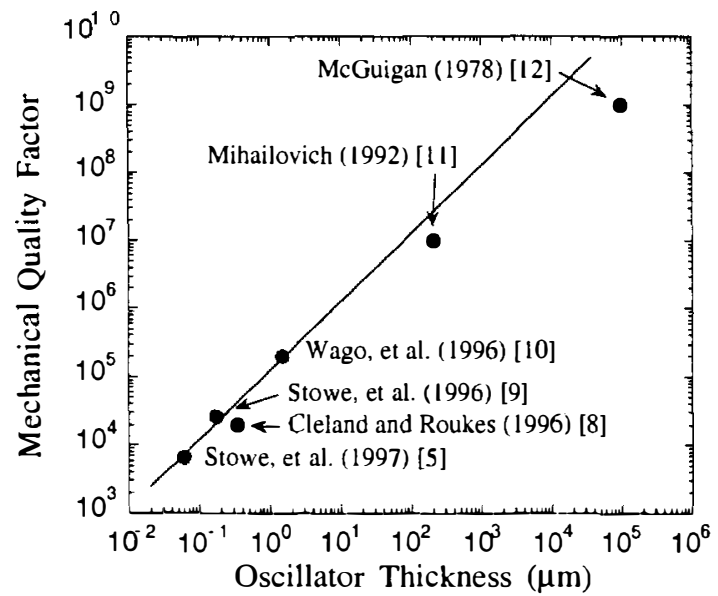

Figure 11. Mechanical quality factor vs. oscillator thickness for single crystal silicon oscillators at $T=4 K$.

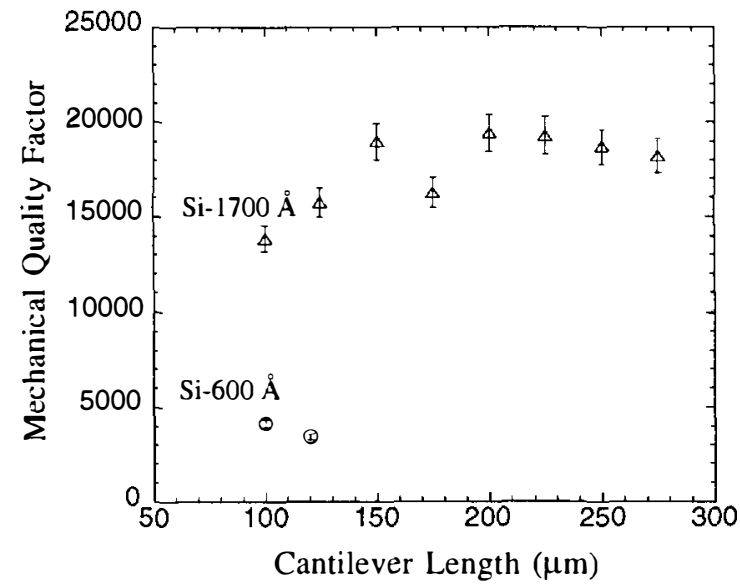

Figure 10. Mechanical quality factor vs. cantilever length for single crystal silicon cantilevers of thickness 600 and $1700 \AA$.

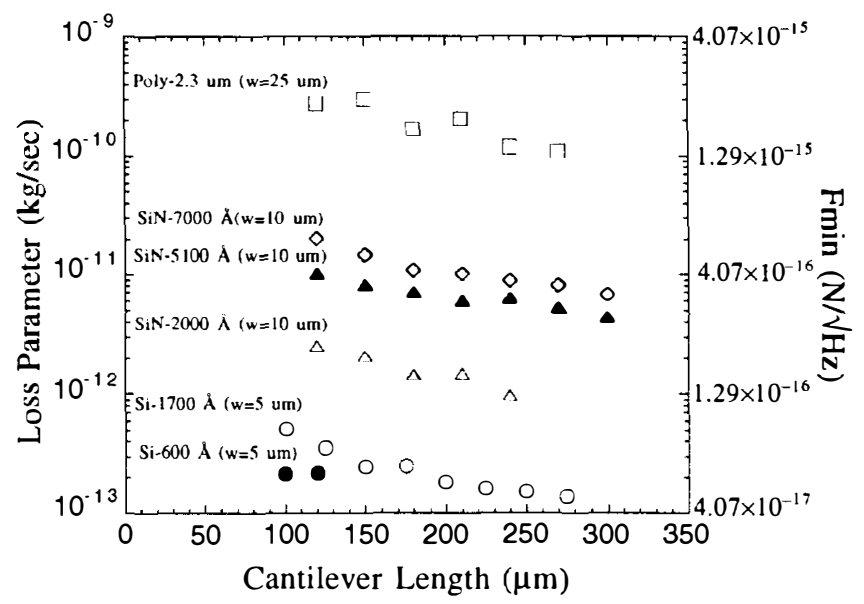

Figure 12. Loss parameter $\gamma=k \omega_{0} Q$ vs. cantilever length comparison plot. Arrays of silicon nitride, polysilicon, and single crystal silicon cantilevers are shown with their thicknesses and widths. The equivalent minimum force detectable is shown on the right for cantilevers at room temperature.

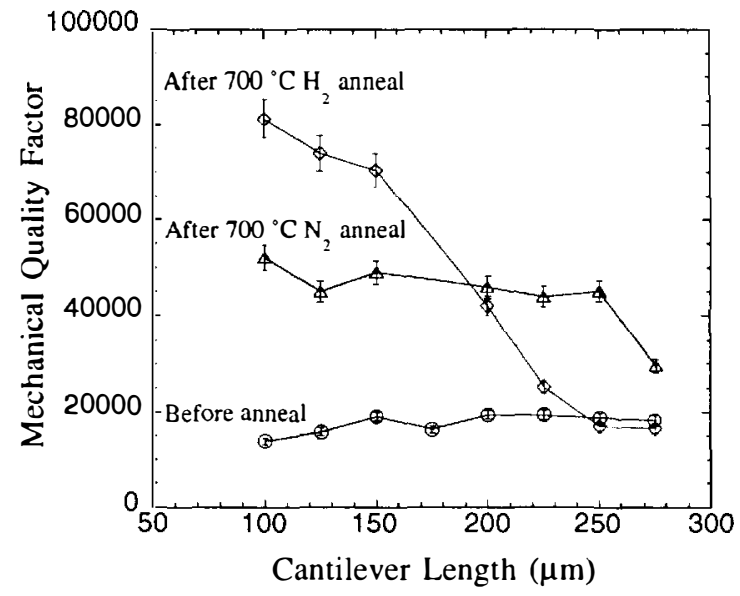

Figure 13. Mechanical quality factor vs. cantilever length for a die of single crystal silicon cantilevers of thickness $1700 \AA$ showing the effects of surface treatments. 
summary plot of the loss parameter $\gamma$ for silicon nitride, polysilicon, and single crystal silicon cantilevers. The equivalent minimum force detectable at room temperature is also shown on Fig. 12 for comparison. A 1700 Å-thick single crystal silicon cantilever of length $275 \mu \mathrm{m}$, width $5 \mu \mathrm{m}$, and $Q$ of $1.8 \times 10^{4}$ has a loss parameter $\gamma=1.4 \times 10^{-13} \mathrm{~kg} / \mathrm{sec}$. This corresponds to a room temperature force noise of $4.8 \times 10^{-17}$ $\mathrm{N} / \sqrt{\mathrm{Hz}}$ or $48 \mathrm{aN} / \sqrt{\mathrm{Hz}}$. Let us now compare this cantilever to a thicker nitride cantilever of approximately the same length. A $5100 \AA$-thick silicon nitride cantilever of length $270 \mu \mathrm{m}$, width $10 \mu \mathrm{m}$, and $Q$ of $1.5 \times 10^{4}$ has a loss parameter $\gamma=5.1 \times 10^{-11}$ $\mathrm{kg} / \mathrm{sec}$ and a force sensitivity of $290 \mathrm{aN} / \mathrm{JHz}$. Thus the silicon cantilever has six times lower equivalent force noise than the thicker silicon nitride cantilever at room temperature. In Fig. 12 we can also see that the relationship between cantilever stiffness and length causes the longer cantilevers to have improved force resolution than the shorter cantilevers. This is expected because longer cantilevers have lower $k / \omega_{0}$ yet have the same approximate $Q$.

\section{SURFACE TREATMENTS}

As seen in Figs. 8 and 11, the mechanical $Q$ of both silicon nitride and single crystal silicon cantilevers are dependent on the cantilever thickness, suggesting surface dominated loss mechanisms. Possible sources of surface loss are adsorbates on the cantilever surface or surface defects created during the cantilever fabrication process. High temperature surface treatments of $1700 \AA$-thick single crystal silicon cantilevers are shown in Fig. 13. A 1 hour $700^{\circ} \mathrm{C}$ anneal in a nitrogen atmosphere increased the $Q$ by a factor of two. Subsequent annealing of the same cantilever array in forming gas (Ar with $4.25 \% \mathrm{H}_{2}$ ) for 1 hour at $700^{\circ} \mathrm{C}$ caused additional increase in the $Q$ for the shorter cantilevers-up to 80,000 . The ability to influence the $Q$ of these cantilevers so dramatically using surface treatments is promising.

\section{CONCLUSIONS}

The goal of this study is to understand the dissipation mechanisms in micromechanical oscillators. For all of the silicon nitride and single crystal silicon cantilevers studied, surface dissipation mechanisms appear to be the dominant source of energy dissipation. For both silicon nitride and single crystal silicon cantilevers a linear $Q$ vs. cantilever thickness behavior has been observed. This behavior is consistent with surface-dominated energy dissipation. Further, preliminary experiments on $700^{\circ} \mathrm{C}$ surface treatments of silicon cantilevers indicates that surface contaminants or defects can be removed via such treatments allowing up to four fold $Q$ increase.

It is clear that further experiments should be carried out. For example, performing experiments in ultra-high vacuum would allow better control over surface characteristics. Also, $Q$ measurements over a broader set of cantilever thicknesses need to be performed to establish a quantitative thickness dependence of the $Q$. Finally, the role of other surface characteristics, such as deposited films, should be explored.

\section{ACKNOWLEDGMENTS}

This work has been supported by the National Science Foundation CAREER Award (ECS-9502046), the National Science Foundation GOALI Award (ECS-9422255), the National Science Foundation Instrumentation for Materials Research Program (DMR 9504099), and the Terman Fellowship.

\section{REFERENCES}

1. T.B. Gabrielson, "Mechanical-Thermal Noise in Micromachined Acoustic and Vibration Sensors", IEEE Transactions of Electron Devices, 40, 5, 903-9 (1993).

2. R.E. Mihailovich and N.C. MacDonald, "Dissipation measurements in vacuum-operated single-crystal silicon microresonators", Sensors and Actuators A, 50, 199-207 (1995).

3. V.B. Braginsky, "Systems with Small Dissipation" (The University of Chicago Press, Chicago, 1985).

4. R.A. Buser and N.F. De Rooij, "Very High $Q$-factor Resonators in Monocrystalline Silicon", Sensors and Actuators, A21-A23, 323-327 (1990).

5. T.D. Stowe, K. Yasumura, T.W. Kenny, D. Botkin, K. Wago, and D. Rugar, "Attonewton force detection using ultrathin silicon cantilevers”, Appl. Phys. Lett., 71, 288-290 (1997).

6. G.T. Mulhern, D.S. Soane, and R.T. Howe, 1993 International Conference on Solid-State Sensors and Actuators: Transducers '93 (Yokohama, Japan, 1993).

7. Polytec P.I., 302 Sensor Head with OVF3001 Controller.

8. A.N. Cleland and M.L. Roukes, "Fabrication of high frequency nanometer scale mechanical resonators from bulk $\mathrm{Si}$ crystals", Appl. Phys. Lett., 69, 2653-5 (1996).

9. T.D. Stowe, K. Yasumura, T.W. Kenny, D. Botkin, K. Wago, and D. Rugar, "Ultrasensitive vertical force probe for magnetic resonance force microscopy", Solid-State Sensor and Actuator Workshop, Hilton Head, South Carolina (1996).

10. K. Wago, O. Züger, R. Kendrick, C.S. Yannoni, and D. Rugar, "Low temperature magnetic resonance force detection", J. Vac. Sci. Technol. B, 14, 2, 1197-201 (1996).

11. R.E. Mihailovich, "Low temperature properties of boron doped silicon”, Phys. Rev. Lett., 68, 3052-5 (1992).

12. D.F. McGuigan, "Measurements of the mechanical $Q$ of single-crystal silicon at low temperatures", J. Low Temp. Phys., 30, 621-9 (1978). 